\title{
Round Blue Cell Tumor arising in the Background of Ameloblastic Fibrosarcoma
}

\author{
Raza Hussain, Usman Hassan, Arif Jamshed, Asif Loya
}

\begin{abstract}
World Health Organization (WHO) blue book of head and neck tumors reports 64 cases of ameloblastic fibrosarcoma and 14 cases of ameloblastic fibrodentinosarcoma/fibro-odontosarcoma. We present a unique case that does not fit into any of the WHO classified odontogenic sarcomas. The patient presented in 2006 with a densely sclerotic lesion arising from the left sphenoid bone, displacing the orbital contents and extending up to the inferior margin of the maxillary sinus. He was treated with left maxillectomy orbital exenteration. He relapsed 6 years later, salvage surgery was unsuccessful and subsequent care was supportive. Histologically the malignant neoplasm showed follicles comprising of peripheral columnar cells lined up in palisaded fashion with hyperchromatic nuclei displaced away from basement membrane. Center of the follicles comprised of spindle-shaped cells resembling stellate reticulum. Intervening areas showed fibrosarcomatous areas. Other areas revealed predominant sheets of round cells having hyperchromatic nuclei, inconspicuous nucleoli and scant cytoplasm. These round cells revealed strong membranous positivity for CD99.
\end{abstract}

Keywords: Ameloblastic carcinoma, Round blue cell tumor, Head and neck.

How to cite this article: Hussain R, Hassan U, Jamshed A, Loya A. Round Blue Cell Tumor arising in the Background of Ameloblastic Fibrosarcoma. Int J Head Neck Surg 2013;4(2): 83-85.

\section{Source of support: Nil}

Conflict of interest: None declared

\section{INTRODUCTION}

Malignant odontogenic tumors are generally rare. Odontogenic sarcomas comprise of ameloblastic fibrosarcoma, ameloblastic fibrodentinosarcoma and ameloblastic fibroodontosarcoma. ${ }^{1}$ WHO blue book of head and neck tumors reports 64 cases of ameloblastic fibrosarcoma and 14 cases of ameloblastic fibrodentinosarcoma/fibro-odontosarcoma. ${ }^{2}$ Ameloblastic fibrosarcoma has locally aggressive behavior with extremly low potential for distant metastasis. Ameloblastic fibrodentinosarcoma/fibro-odontosarcoma has better prognosis as compared to other jaw sarcomas. ${ }^{3}$ We present a unique case that does not fit into any of the WHO classified odontogenic sarcomas and label it as round blue cell tumor arising in the background of ameloblastic fibrosarcoma.

\section{CASE REPORT}

A 30 years old male presented with a 1 year history of leftsided facial swelling in 2006. Computed tomographic (CT) scan showed a densely sclerotic lesion arising from the left sphenoid bone, displacing the orbital contents and extending up to the inferior margin of the maxillary sinus (Fig. 1). Patient underwent left maxillectomy and orbital exenteration. The pathology specimen was oriented with single stitch on superior margin, double stitches on medial margin, triple stitches on optic nerve resection margin, four stitches on temporalis margin and five stitches on zygomatic arch margin. The whole of the specimen measured $8 \times 5 \times 3 \mathrm{~cm}$. Serial sectioning revealed a well- circumscribed tumor measuring $6 \times 4 \times 3 \mathrm{~cm}$ located at a distance of $4 \mathrm{~cm}$ from superior margin, $0.2 \mathrm{~cm}$ from inferior margin, $1.5 \mathrm{~cm}$ from medial margin, 0.5 $\mathrm{cm}$ from lateral margin, $0.3 \mathrm{~cm}$ from posterior margin and 2 $\mathrm{cm}$ each from temporalis and zygomatic resection margins. Eyeball was not involved by the tumor. Cut surface of the tumor was gray white, firm and multilobulated. Histological sections revealed a malignant neoplasm showing follicles comprising peripheral columnar cells lined up in palisaded fashion. Nuclei were hyperchromatic and displaced away from basement membrane. Center of the follicles comprised of spindle-shaped cells resembling stellate reticulum (Fig. 2). Intervening areas showed fibrosarcomatous areas comprising proliferated spindle cells showing moderate atypia and atypical mitoses (Fig. 3). Other areas revealed predominant sheets of round cells having hyperchromatic nuclei, inconspicuous nucleoli and scant cytoplasm (Fig. 4). These round cells revealed strong membranous positivity for CD 99 (Fig. 5). We labeled this case as round blue cell tumor

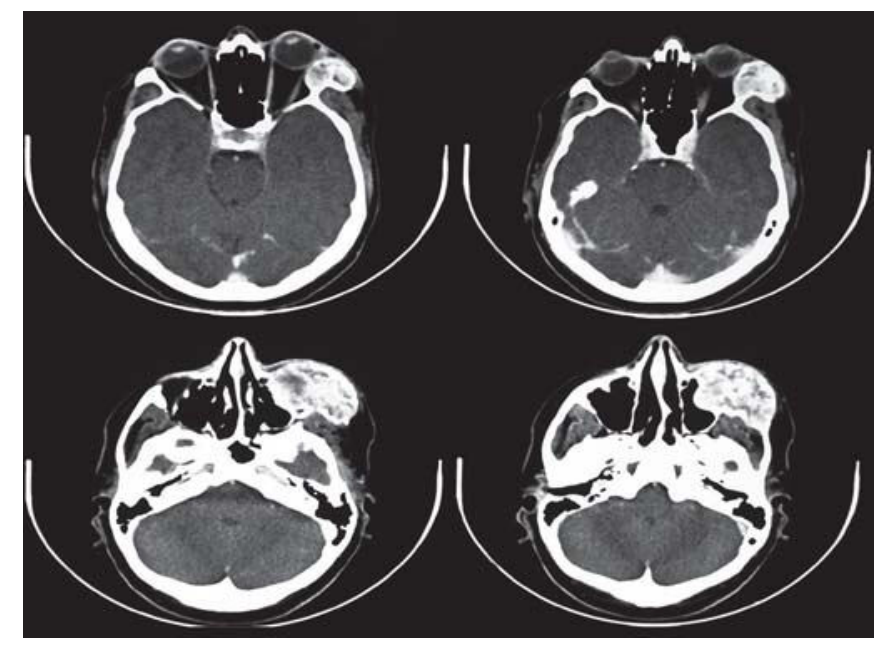

Fig. 1: CT scan showing a densely sclerotic lesion arising from the left sphenoid bone, displacing the orbital contents and extending to the left maxillary sinus 
arising in the background of ameloblastic fibrosarcoma. Postoperative $\mathrm{CT}$ scan did not show any residual tumor and radiation therapy $60 \mathrm{~Gy}$ in 30 fractions were delivered to the operative bed. He remained symptom free for 6 years and re-presented with a large recurrent mass involving ethmoid sinuses. Biopsy of the recurrent lesion again revealed round blue cell tumor arising in the background of ameloblastic fibrosarcoma.

\section{DISCUSSION}

Odontogenic sarcomas are classified as ameloblastic fibrosarcomas and ameloblastic fibrodentinosarcoma and fibro-odontosarcomas. They usually occur in a wide age range (3-89 years) with median age in the third decade. Most of ameloblastic fibrosarcomas occur in mandible. ${ }^{4}$ Usually these sarcomas are locally aggressive. These have better prognosis than other jaw sarcomas and are generally regarded as low grade sarcomas. Ameloblastic sarcoma was first reported by

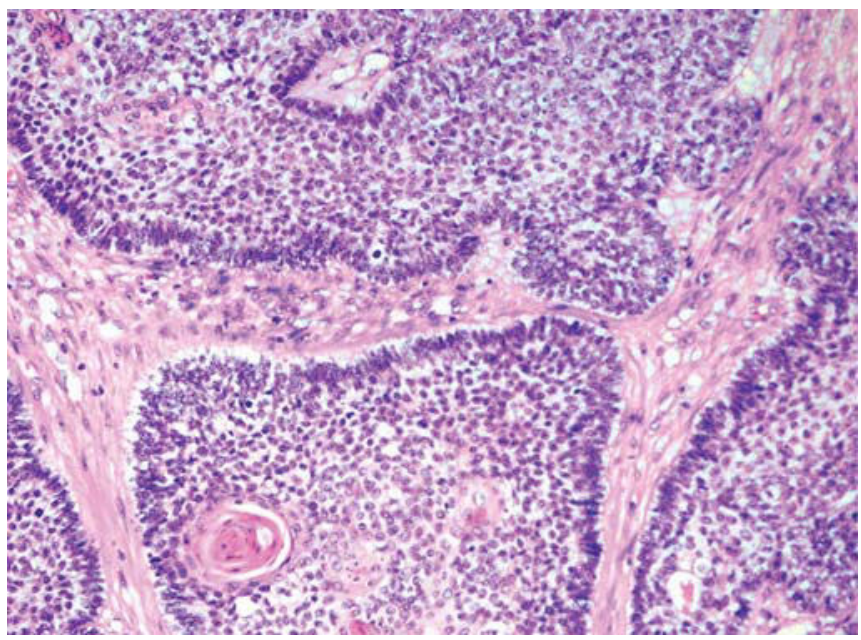

Fig. 2: Follicles comprising peripheral columnar cells lined up in palisaded fashion. Center of the follicles comprised of spindleshaped cells resembling stellate reticulum

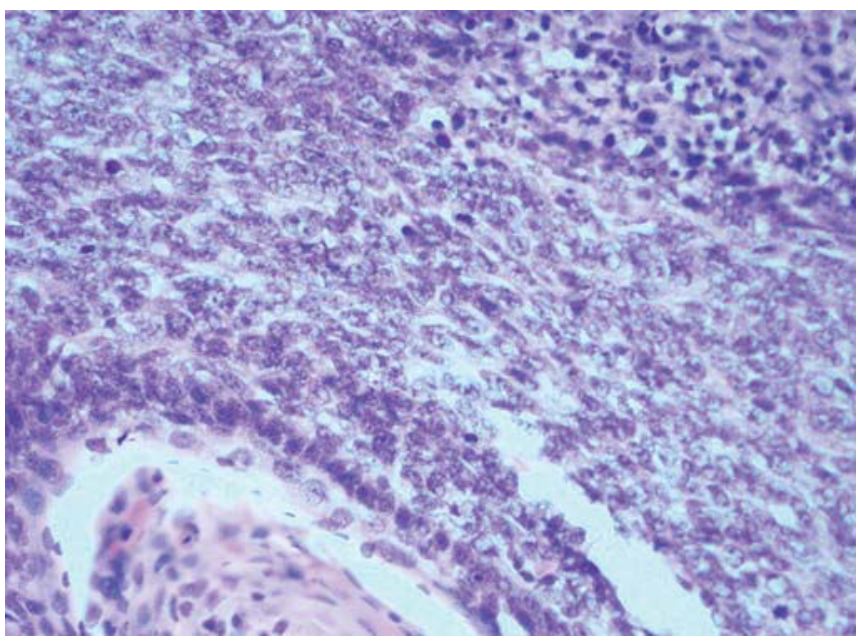

Fig. 3: Fibrosarcomatous areas comprising proliferated spindle cells showing moderate atypia and atypical mitoses
Heath in $1887 .{ }^{5}$ It has then been reported by many authors over a period of many years from all around the world. Previously ameloblastic sarcoma had been used as a general term to include all types of odontogenic sarcomas. Later on it was further classified as ameloblastic fibrosarcomas and ameloblastic fibrodentino- and fibro-odontosarcomas. This segregation was based on the presence or absence of dentin or dentin or enamel in the lesion. Ameloblstic fibrosarcoma has been reported by Chromette in $1982,{ }^{6}$ Goldstein G in $1976,{ }^{7}$ Pinborg in $1960,{ }^{8}$ Kousar in $2009,{ }^{9}$ DemoorGoldschmidt in $2012^{1}$ and many other authors. About 14 cases of fibrodentino- and odontosarcomas have been reported. ${ }^{2,3}$ In majority of the cases reported patients were middle aged and most common location was mandible followed by maxilla. Most of the cases showed locally aggressive behavior. Surgical treatment was curative in majority of the cases. Only one case showed metastasis to mediastinal nodes.

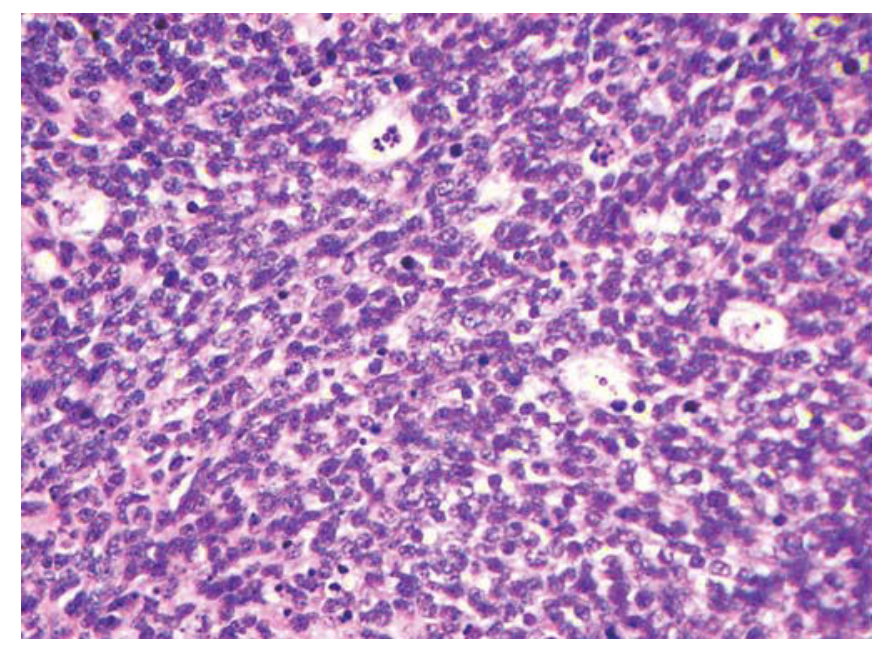

Fig. 4: Predominant sheets of round cells having hyperchromatic nuclei, inconspicuous nucleoli and scant cytoplasm

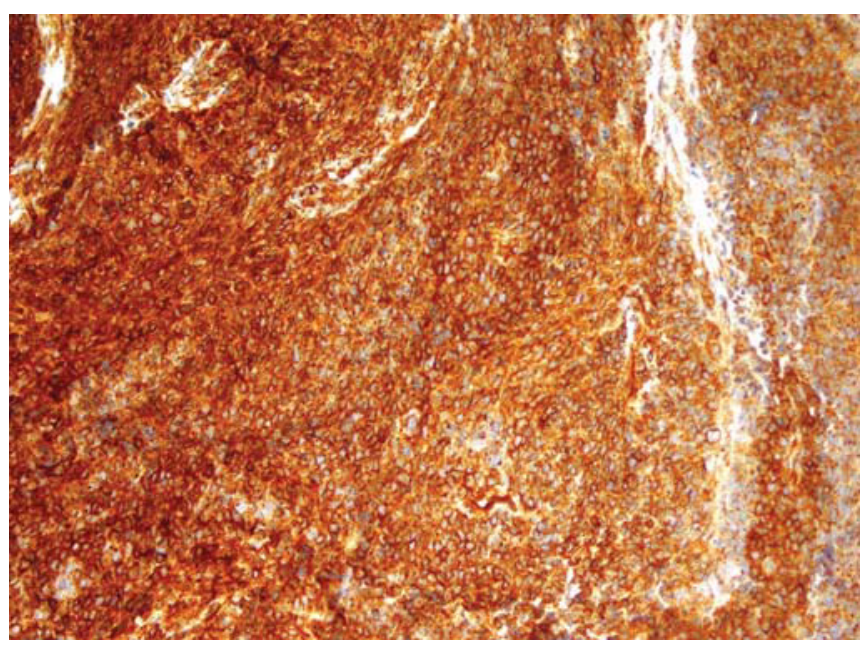

Fig. 5: Round cells revealed strong membranous positivity for CD99 
Our case is unique due to the fact that it involved maxilla and it was different from ameloblastic fibrosarcomas and ameloblastic fibrodentinosarcoma fibro-odantosarcomas. This tumor had a component of ameloblastoma and the other components comprising fibrosarcoma and round blue cell tumor component. The latter component had cells with increased nuclear to cytoplasmic ratio, hyperchromatic nuclei and frequent atypical mitoses. These cells were positive for CD99. This tumor behaved aggressively. It recurred after a period of 6 years despite complete surgical resection and radiotherapy. We labeled our case as round blue cell tumor arising in a background of ameloblastic fibrosarcoma. We have not been able to find such a case in the literature.

Usually, odontogenic tumors are low grade sarcomas but if round blue cell component occurs in background of ameloblastoma it can behave in an aggressive fashion.

\section{REFERENCES}

1. Demoor-Goldschmidt C, Minard-Colin V, Cassagneau E, et al. Ameloblastic fibrosarcoma of the mandible: Report of 2 chemosensitive pediatric cases. J Pediatr Hematol Oncol 2012 Mar;34(2):e72-76.

2. Bregni RC, Taylor AM, Garcia AM. Ameloblastic fibrosarcoma of the mandible: Report of two cases and review of the literature. J Oral Pathol Med 2001;30:316-20.

3. Takeda Y. Ameloblastic fibrosarcoma and related lesions: Current pathologic concept. Oral Oncol 1999;35:535-40.

4. Guthikonda B, Hanna EY, Skoracki RJ, Prabhu SS. Ameloblastic fibrosarcoma involving the anterior and middle skull base with intradural extension. J Craniofac Surg 2009 Nov;20(6):2087-90.

5. Heath C. Five cases of tumours of jaws treated by excision. BMJ 1887;1:777-79.
6. Chomette G, Auriol M, Guilbert F, Ginisty D, Akil N. Ameloblastic fibrosarcoma. A clinical and anatomico-pathological study of three cases. Histoenzymological and ultrastructural data. Arch Anat Cytol Pathol 1982;30:172-78.

7. Goldstein G, Parker FP, High GSF. Ameloblastic sarcoma: Pathogenesis and treatment with chemotherapy. Cancer 1976;37: 1673-78.

8. Pinborg JJ. Ameloblastic sarcoma in the maxilla: Report of a case. Cancer 1960;13:917-20.

9. Kousar A, Hosein MM, Ahmed Z, Minhas K. Rapid sarcomatous transformation of an ameloblastic fibroma of the mandible: Case report and literature review. Oral Surg Oral Med Oral Pathol Oral Radiol Endod 2009 Sep;108(3):e80-85.

\section{ABOUT THE AUTHORS}

\section{Raza Hussain}

Consultant, Department of Surgery, Shaukat Khanum Memorial Cancer Hospital and Research Centre, Lahore, Punjab, Pakistan

\section{Usman Hassan}

Resident, Department of Pathology, Shaukat Khanum Memorial Cancer Hospital and Research Centre, Lahore, Punjab, Pakistan

\section{Arif Jamshed (Corresponding Author)}

Consultant, Department of Radiation Oncology, Shaukat Khanum Memorial Cancer Hospital and Research Centre, Lahore, Punjab Pakistan, e-mail: jamshedarif@hotmail.com

\section{Asif Loya}

Consultant, Department of Pathology, Shaukat Khanum Memorial Cancer Hospital and Research Centre, Lahore, Punjab, Pakistan 